\title{
Knowledge of and willingness to take pre-exposure prophylaxis among men who have sex with men in Israel
}

Mor Zohar ${ }^{1,2^{*}}$, Shilo Guy ${ }^{3}$ and Levy Itzchak ${ }^{4,5}$

\begin{abstract}
Background: Oral pre-exposure prophylaxis (PrEP) with tenofovir disoproxil fumarate and emtricitabine (TDF/FTC) has been found to reduce viral acquisition among HIV-negative MSM. This cross-sectional study was conducted before pre-exposure prophylaxis (PrEP) licensure in Israel, and aimed to compare men who have sex with men (MSM) who had heard of PrEP with those who had not, as well as MSM willing to take PrEP with those who were hesitant or not willing to take PrEP.

Methods: HIV-negative MSM responded anonymously to questionnaires in 2017 regarding their knowledge of and willingness to take PrEP, prior use of PrEP and post-exposure prophylaxis (PEP), and their sexual behaviors.

Results: Among 1705 participants, 1431 (83.9\%) had heard about PrEP. They were older and more often reported being Jewish, having an academic degree, self-identifying as gay/bisexual, being tested for HIV in the last year, participating in group sex, using alcohol or drugs before or during sex, and having prior use of PrEP/PEP compared with MSM who had not heard about PrEP. A total of 760 (44.8\%) participants indicated that they would consider taking PrEP, 567 (33.5\%) maybe would consider taking PrEP, and 367 (21.7\%) would not take PrEP. Those who were willing to take PrEP had a lower level of education, were involved in high-risk sexual behaviors, used alcohol or drugs before or during sex, and had previously used PrEP/PEP compared with participants who maybe would consider taking or would not take PrEP. When participants were asked to indicate if they were willing to take PrEP at different potential efficacies and costs, the willingness to using PrEP increased with the potential efficacy of the drug and adversely related to its cost.
\end{abstract}

Conclusions: PrEP awareness was high, and $44.8 \%$ indicated willingness to take PrEP, especially those who reported high-risk sexual behaviors. This supports the current policy in Israel to allow PrEP to MSM who are at high-risk. In order to maintain a high level of PrEP-adherence, physicians should consider structural barriers, such as negative stigma of being promiscuous, lack of perceived HIV-risk, difficulties in accessing clinics or paying for PrEP, inability to follow-up or low tolerability of the medication.

Keywords: Gay men, HIV, Post-exposure prophylaxis, Sexual behavior

*Correspondence: zohar.mor@telaviv.health.gov.il

${ }^{1}$ Tel Aviv Department of Health, Ministry of Health, 12 Ha'arba'a Street, Tel Aviv, Israel

Full list of author information is available at the end of the article

\section{Background}

Men who have sex with men (MSM) are affected disproportionately by HIV in Israel and other high-income countries [1]. A wide array of efforts, including behavioral interventions and encouraging frequent HIV testing and the use of barrier methods such as condoms, have been employed among MSM to decrease their exposure original author(s) and the source, provide a link to the Creative Commons licence, and indicate if changes were made. The images or other third party material in this article are included in the article's Creative Commons licence, unless indicated otherwise in a credit line to the material. If material is not included in the article's Creative Commons licence and your intended use is not permitted by statutory regulation or exceeds the permitted use, you will need to obtain permission directly from the copyright holder. To view a copy of this licence, visit http://creativecommons.org/licenses/by/4.0/. The Creative Commons Public Domain Dedication waiver (http://creativeco mmons.org/publicdomain/zero/1.0/) applies to the data made available in this article, unless otherwise stated in a credit line to the data. 
to sexual risks [2]. Following the introduction of antiretroviral treatment (ART), scientific evidence has demonstrated the benefits of treatment as prevention and that the reduction of patients' HIV viral load decreases the risk of viral transmission in susceptible social/sexual networks [3]. Recently, the use of oral pre-exposure prophylaxis (PrEP) with combined tenofovir disoproxil fumarate $300 \mathrm{mg}$ and emtricitabine $200 \mathrm{mg}$ (TDF/FTC) has been found to reduce viral acquisition among HIV-negative MSM [4].

This biomedical breakthrough of using PrEP to reduce significantly the risk of acquiring HIV was confirmed by the US Food and Drug Administration's approval of TDF/ FTC for PrEP in HIV-negative adults at high risk [5] and the release of new guidelines by the World Health Organization [6] and the U.S. Centers for Disease Control and Prevention [7]. Daily use of PrEP was approved in Israel in September 2017 for a subset of MSM who engage in high-risk sexual behavior [8], while efforts to encourage this population to use condoms have continued.

The success of PrEP depends on the availability of the drug, convenience of use, and its acceptability by the community. A recent meta-analysis reported an estimate of PrEP acceptability of $57.8 \%$ among MSM [9]. In Israel, data are lacking regarding MSM's knowledge of PrEP and their willingness to use PrEP, although the rate of HIV acquisition among MSM is disproportionately high. Among all males diagnosed with HIV in Israel between 1981 and 2017, the proportion of MSM was 38.6\% [10].

The purpose of this study was to compare the number of MSM who had heard about PrEP with those who had not heard about PrEP. In addition, we aimed to compare MSM who were willing to take PrEP with those who maybe would take or were not willing to take PrEP. This study was performed prior to the licensure of PrEP in Israel to allow policy stakeholders and community leaders to estimate the potential number of MSM who are interested in taking PrEP, and assess their behavioral characteristics, as an important step toward increasing PrEP utilization.

\section{Methods}

\section{Participants and procedures}

This cross-sectional study included HIV-negative MSM recruited between May and July 2017 through geosocialnetworking smartphone applications (Grindr, Atraf), which are commonly used by MSM to meet potential sexual partners, as well as through virtual social networks (Facebook). Application users received pop-up advertisements with a link to the survey. After signing informed consent forms, participants were asked to reply anonymously to a set of 52 questions regarding their demographic characteristics, whether or not they had heard about PrEP, prior PrEP or post-exposure prophylaxis (PEP) use, and their sexual behaviors during the last year (Additnal file 1: Appendix). In order to encourage participants to respond to the questionnaire, a raffle was performed, awarding the winners with annual gym subscriptions.

\section{Measures}

High-risk sexual behavior was measured by the HIV Incidence Risk Index for MSM (HIRI-MSM) score [11], which includes age and the following criteria concerning the last six months: the total number of male sex partners and HIV-positive partners, the number of condomless receptive anal sex encounters with any HIV status partner, the number of encounters that included insertive anal sex with an HIV-positive partner, and also the use of poppers or amphetamines. Participants were scored between 0 and 47, and a score of 10 or greater suggests high sexual risk.

\section{Variables}

Dependent variables for this study were PrEP awareness, which was assessed by asking participants if they had heard about PrEP (yes/no), and willingness to take PrEP, which was assessed by asking participants whether they would consider taking PrEP (yes/maybe/no). Independent variables include demographic characteristics, sexual behavior during the last year, previous use of PEP or PrEP, and their attitudes towards potential sexual risks.

\section{Statistical analysis}

Independent variables were compared using Chi-square or Student's $t$-tests for categorical and continuous variables, respectively. Variables whose $p$ values were less than $5 \%$ in the univariate analyses were included in the multivariate analysis to identify attributes predicting willingness to take PrEP.

\section{Results}

Between May and July 2017, 2645 responders started answering the questionnaire: 2472 (93.5\%) were recruited through smartphone applications and 173 (6.5\%) through Facebook. Of these, 914 did not complete the questionnaire, 12 did not have sex in the last year, nine had sex only with women, and five provided inconsistent responses. The average age of the 1705 MSM who met the inclusion criteria was 32 years (range 18-77). Among all participants, 1561 (91.5\%) were born in Israel, 1594 were Jews (93.5\%), 957 (56.1\%) had an academic degree, $1423(83.5 \%)$ self-identified as gay or bisexual, and 409 (23.9\%) had a steady partner.

Overall, 1431 (83.9\%) had previously heard about PrEP (Table 1). Those who had heard about PrEP were older 
Table 1 Comparison of characteristics of MSM who had heard about or had not heard about pre-exposure prophylaxis

\begin{tabular}{|c|c|c|c|}
\hline Characteristics & $\begin{array}{l}\text { Heard of PrEP } \\
\mathrm{N}=1431(\%)\end{array}$ & $\begin{array}{l}\text { Had not heard of PrEP } \\
\mathrm{N}=274(\%)\end{array}$ & $P$ \\
\hline \multicolumn{4}{|l|}{ Demographics } \\
\hline Age in years $\pm S D$ & $33.7 \pm 8.7$ & $32.1 \pm 10.8$ & 0.02 \\
\hline Israeli born & $1315(91.8)$ & $246(89.7)$ & 0.2 \\
\hline Jewish & $1350(95.1)$ & $244(90.4)$ & 0.003 \\
\hline Academic degree & $842(58.9)$ & $115(42.0)$ & $<0.001$ \\
\hline Self-definition as a gay or bisexual ma & $1232(86.2)$ & $191(71.5)$ & $<0.001$ \\
\hline \multicolumn{4}{|l|}{ Sexual behaviors in the past year } \\
\hline HIV test in the past year & $1358(95.0)$ & $242(89.3)$ & $<0.001$ \\
\hline Sex with both men and women & $127(10.2)$ & $54(19.9)$ & $<0.001$ \\
\hline Age at first anal sex & $18.3 \pm 4.6$ & $18.3 \pm 5.1$ & 0.9 \\
\hline Current steady partner & $347(24.3)$ & $62(23.3)$ & 0.7 \\
\hline Open steady relationships & $263(73.8)$ & $31(50.0)$ & $<0.001$ \\
\hline CAI with partner whose HIV status was unknown/discordant & $573(40.6)$ & $111(41.7)$ & 0.7 \\
\hline Prefers receptive anal sex & $812(57.2)$ & $145(56.0)$ & 0.7 \\
\hline Participation in group sex & $563(40.5)$ & $88(33.5)$ & 0.04 \\
\hline Special sexual repertoire ${ }^{a}$ & $355(27.5)$ & $58(26.4)$ & 0.6 \\
\hline Sexual risk behavior score ${ }^{b}$ & $17.0 \pm 9.5$ & $16.6 \pm 9.5$ & 0.5 \\
\hline Previous STD diagnosis & $279(21.5)$ & $44(18.9)$ & 0.4 \\
\hline Alcohol or drug use before or during sex & $901(69.4)$ & $130(56.3)$ & $<0.001$ \\
\hline \multicolumn{4}{|l|}{ PrEP/PEP use } \\
\hline Previous use of PEP & $84(5.8)$ & $1(0.4)$ & $<0.001$ \\
\hline Previous use of PrEP & $236(16.5)$ & $19(6.9)$ & $<0.001$ \\
\hline \multicolumn{4}{|l|}{ Attitudes } \\
\hline My sexual behavior is riskier than that of my friends & $223(15.6)$ & $50(19.0)$ & 0.2 \\
\hline I am generally more horny than my friends & $515(36.4)$ & $97(36.3)$ & 0.9 \\
\hline $\begin{array}{l}\text { I can have CAI with an HIV-positive partner who takes antiretroviral treat- } \\
\text { ment }\end{array}$ & $453(32.3)$ & $45(17.4)$ & $<0.001$ \\
\hline
\end{tabular}

CAl condomless anal intercourse, PEP post-exposure prophylaxis, PrEP pre-exposure prophylaxis, SD standard deviation, STD sexually transmitted disease

a Urination, fisting, sado, bondage

${ }^{\mathrm{b}}$ HIV Incidence Risk Index for MSM (HIRI-MSM) score

and more often reported being Jewish, having an academic degree, and self-identifying as gay or bisexual compared with MSM who had not heard about PrEP. Those who had heard about PrEP more often reported having been tested for HIV in the last year, participating in group sex, and using alcohol or drugs before or during sex, and less often reported having concurrent sex with women. In addition, those who had heard about PrEP more often reported previous use of PrEP or PEP and that they would have condomless anal intercourse with an HIV-positive partner who is taking ART.

Of the 1694 participants who responded regarding their willingness to take PrEP, 760 (44.8\%) indicated that they would consider taking PrEP, 567 (33.5\%) maybe would consider taking PrEP, and 367 (21.7\%) would not consider taking PrEP (Table 2). Those who were willing to take PrEP were less likely to have an academic degree, but more likely to have been tested for HIV in the past year, report earlier anal sex, have condomless anal intercourse with a partner whose HIV status was unknown or discordant, to prefer receptive anal intercourse, participate in group sex and other sexual repertoire, have a higher HIRI-MSM risk score, use alcohol or drugs before or during sex, and to have previously used PrEP or PEP compared with participants who maybe would take PrEP and those who would not consider taking it. MSM who were willing to take PrEP reported taking more sexual risks and having a greater sexual appetite than their friends. They also reported that they would have condomless anal intercourse with an HIV-positive partner who is taking ART. MSM who were willing to take PrEP were less likely to be diagnosed with an STD in the past year than those who did not consider taking PrEP. In the multivariate analysis, a lower level of education, having 
Table 2 Comparison of characteristics of MSM who would consider taking PrEP with those who maybe or would not consider taking PrEP

\begin{tabular}{|c|c|c|c|c|c|c|}
\hline \multicolumn{5}{|l|}{ Univariate } & \multicolumn{2}{|l|}{ Multivariate $\wedge$} \\
\hline Characteristics & $\begin{array}{l}\text { Would } \\
\text { consider } \\
\text { taking PrEP } \\
N=760 \\
(44.8 \%)\end{array}$ & $\begin{array}{l}\text { Maybe would } \\
\text { consider taking } \\
\text { PrEP } \\
N=567(33.5 \%)\end{array}$ & $\begin{array}{l}\text { Would not } \\
\text { consider taking } \\
\text { PrEP } \\
N=367(21.7 \%)\end{array}$ & $P$ & OR $(95 \% \mathrm{Cl})$ & $P$ \\
\hline \multicolumn{7}{|l|}{ Demographics } \\
\hline Age, years $\pm S D$ & $33.8 \pm 9.4$ & $32.7 \pm 8.7$ & $33.6 \pm 8.8$ & 0.09 & & \\
\hline Israeli born & $689(90.0)$ & $520(91.7)$ & $341(93.4)$ & 0.4 & & \\
\hline Jewish & $713(94.4)$ & $525(93.4)$ & $346(94.6)$ & 0.5 & & \\
\hline Academic degree & $361(47.5)$ & $322(56.9)$ & $230(63.0)$ & 0.005 & $0.8(0.6-0.9)$ & 0.02 \\
\hline Self-definition as a gay or bisexual man & $638(84.1)$ & $466(82.2)$ & $309(84.6)$ & 0.5 & & \\
\hline \multicolumn{7}{|l|}{ Sexual behaviors in the past year } \\
\hline HIV test in the past year & $729(96.0)$ & $530(93.6)$ & $331(90.5)$ & 0.001 & $1.5(0.9-2.3)$ & 0.1 \\
\hline Sex with both men and women & $79(10.5)$ & $61(10.8)$ & $40(10.9)$ & 0.9 & & \\
\hline Age at first anal sex & $17.8 \pm 4.9$ & $18.3 \pm 4.3$ & $19.1 \pm 4.8$ & $<0.001$ & $1.0(0.9-1.1)$ & 0.6 \\
\hline Current steady partner & $182(24.0)$ & $131(23.4)$ & $93(25.8)$ & 0.7 & & \\
\hline Open steady relationships & $142(78.1)$ & $85(64.9)$ & $62(66.7)$ & 0.02 & & \\
\hline $\begin{array}{l}\text { CAl with partner whose HIV status was unknown or } \\
\text { discordant }\end{array}$ & $403(54.6)$ & $206(36.8)$ & $72(20.3)$ & $<0.001$ & $3.7(2.5-5.6)$ & 0.01 \\
\hline Prefers receptive anal sex & $458(60.9)$ & $183(32.3)$ & $202(56.4)$ & $<0.001$ & $1.4(1.1-1.7)$ & 0.04 \\
\hline Participation in group sex & $357(51.5)$ & $107(21.1)$ & $115(34.5)$ & $<0.001$ & $1.4(1.1-1.8)$ & 0.01 \\
\hline Special sexual repertoire ${ }^{a}$ & $225(34.0)$ & 65 (12.9) & $77(26.4)$ & $<0.001$ & $1.3(1.2-1.4)$ & 0.04 \\
\hline Sexual risk behavior score ${ }^{b}$ & $19.9 \pm 10.1$ & $16 \pm 8.5$ & $12.6 \pm 7.5$ & $<0.001$ & $1.2(1.1-1.4)$ & 0.04 \\
\hline Previous STD diagnosis & $167(24.5)$ & $51(10.2)$ & $105(31.8)$ & $<0.001$ & $1.0(0.8-1.3)$ & 0.9 \\
\hline Alcohol or drug use before or during sex & $519(75.0)$ & $327(65.6)$ & $185(57.0)$ & 0.005 & $1.4(1.2-1.8)$ & 0.002 \\
\hline \multicolumn{7}{|l|}{ PEP/PrEP use } \\
\hline Previous use of PrEP & $156(20.5)$ & $35(6.1)$ & $64(17.3)$ & $<0.001$ & $1.5(1.1-2.0)$ & 0.02 \\
\hline $\begin{array}{l}\text { Previous use of PEP } \\
\text { Did not use PEP }\end{array}$ & $81(10.7)$ & $4(0.7)$ & $0(0)$ & $<0.001$ & $13.9(5.0-39.2)$ & $<0.001$ \\
\hline \multicolumn{7}{|l|}{ Attitudes } \\
\hline My sexual behavior is riskier than that of my friends & $168(22.3)$ & $67(12.0)$ & $38(11.2)$ & $<0.001$ & $1.7(1.3-2.3)$ & $<0.001$ \\
\hline I am generally more horny than my friends & $296(40.0)$ & $208(37.6)$ & $108(30.4)$ & 0.008 & $1.1(1.0-2.3)$ & 0.4 \\
\hline $\begin{array}{l}\text { I can have CAI with an HIV-positive partner who takes } \\
\text { antiretroviral treatment }\end{array}$ & $452(61.3)$ & $29(5.3)$ & $16(4.5)$ & $<0.001$ & & \\
\hline
\end{tabular}

CAl condomless anal intercourse, PEP post-exposure prophylaxis, PrEP pre-exposure prophylaxis, SD standard deviation, STD sexually transmitted disease

${ }^{\wedge}$ Would versus maybe/would not consider taking PrEP

a Urination, fisting, sado, bondage

${ }^{\mathrm{b}}$ HIV Incidence Risk Index for MSM (HIRI-MSM) score

condomless anal intercourse with a partner whose HIV status was unknown or discordant, preferring receptive anal sex, participating in group sex and other sexual repertoire, having a higher HIRI-MSM risk score, using alcohol or drugs before or during sex, previously using PrEP or PEP, and taking more sexual risks were associated with willingness to take PrEP.

Of all 1431 who had heard about PrEP, the majority $(\mathrm{N}=779,54.4 \%)$ had learned about PrEP from the Internet and only a few $(\mathrm{N}=24,1.7 \%)$ through their physician.
The majority of participants $(\mathrm{N}=1046,73.0 \%)$ preferred the PrEP regimen to be provided by their family physician, followed by the AIDS Task Force (the most significant AIDS-related non-governmental organization in Israel, $\mathrm{N}=520,36.4 \%)$ or by AIDS treatment centers in hospitals $(\mathrm{N}=307,21.5 \%)$.

Previous use of PrEP was reported by 255 (14.9\%) participants. Of these, 129 (50.6\%) bought it online, 69 (27.1\%) were prescribed PEP but ultimately used it as PrEP, and $57(22.4 \%)$ received the drug from an 
HIV-positive friend. Previous use of PEP was reported by $85(5.0 \%)$ participants. Of these, $69(81.2 \%)$ were prescribed PEP more than once and 46 (66.7\%) were advised to take PEP and started the therapy, but did not complete the monthly course.

The willingness of study participants to use PrEP increased with the potential efficacy of the drug to prevent HIV infection, as it was presented in the questionnaire, and adversely related to its various costs (Table 3 ). More than half were willing to use PrEP if the efficacy had been $>95 \%$ at a monthly cost of 500 NIS ( 120 Euro).

\section{Discussion}

Among all study participants, $83.9 \%$ had heard about PrEP. Of all the 1694 participants who responded regarding their willingness to take PrEP, $44.8 \%$ reported that they were willing to take PrEP, and 33.5\% indicated that they maybe would consider taking it. These figures are comparable with studies conducted in other highincome countries, showing 48\% willingness in the United Kingdom [12], 55\% in Canada [13], 65\% in Germany [14]-depending on the drug's availability, cost, and the time and methods of the study.

In our study, participants who were willing to take PrEP were involved in higher-risk sexual behaviors compared with those who maybe would consider taking PrEP and those who would not consider taking PrEP, as reported elsewhere [15]. As $40.1 \%$ of all study participants reported that they had engaged in condomless anal sex with a partner whose HIV status was unknown or discordant in the last year, PrEP is a valuable risk management strategy among those men. Although PrEP has successfully prevented HIV transmission, its availability may lead to increased risk behaviors, which may result in possible exposure to STDs [16]. However, MSM who access PrEP are under continuous medical supervision. Providers who prescribe PrEP have the opportunity to weigh the sexual health of MSM against their pleasureseeking behavior by supporting PrEP adherence, maintaining continued dialogue related to behavioral risk reduction, and recommending routine counseling and testing for STDs, thus limiting further transmission [17].
Although PrEP was not available in Israel when this study was conducted, $15 \%$ of the participants had used off-label PrEP at least once and approximately half of those purchased the drug online, making it likely that they have not received appropriate and regular medical check-ups. Although this high level of awareness could be viewed as a proxy for higher sexual health literacy or greater access to sexual health information, these drugs have not been supplied by a registered pharmacy and are not regulated. Thus, their pharmacological qualities might differ from those of the licensed drug and, consequently, may not provide complete protection.

Previous use of PEP was reported by $\sim 5 \%$ of the participants in this study. Many of these users sought PEP from a medical care clinic, asking for it due to an alleged incident of potential exposure, but actually intending to use it as PrEP. The availability of PrEP in Israel will probably make this practice unnecessary. This use of PrEP before its licensure in Israel was high compared to the results of the European Men-Who-Have-Sex-WithMen Internet Survey (EMIS-2017) study performed in 2017 Europe and Israel, and the average use was 3.0\% among the total sample of $~ 173,000$ MSM [18].

More than $80 \%$ of the participants in this study had heard about PrEP and $45 \%$ reported that they were willing to take the drug. The high number of men who would consider taking PrEP may be informative to medical insurers, healthcare providers, and drug suppliers in evaluating future interest and estimating PrEP uptake. In order to assess the potential number of PrEP users, we used an estimation of 94,176 (95\% CI 92,562-96,063) self-identified Jewish gay/bisexual men aged 18-44 years living in Israel [19]. Previous research has found that high-risk MSM were twice as likely to respond to convenience surveys and report more risk behaviors compared with probability-based surveys [20]. In order to adjust for over-reporting, we applied a $50 \%$ correction factor to the proportion of participants in this study who reported that they would consider taking PrEP (22.4\%), thus we estimated that 21,095 (95\% CI 20,733-21,518) MSM will potentially be interested in using PrEP in Israel.

Table 3 Willingness to take PrEP among those who have heard about the prevention, by success of the technology and its price

\begin{tabular}{llll}
\hline & Want PrEP & Do not want PrEP & $P$ \\
\hline If PrEP is 80\% successful & $401(60.2)$ & $174(23.1)$ & $<0.001$ \\
If PrEP is 90\% successful & $575(86.1)$ & $270(35.7)$ & $<0.001$ \\
If PrEP is 95\% successful and costs 1000 NIS & $183(27.5)$ & $79(10.5)$ & $<0.001$ \\
If PrEP is 95\% successful and costs 500 NIS & $356(53.7)$ & $163(21.7)$ & $<0.01$ \\
If PrEP requires monthly visits + blood tests & $449(67.9)$ & $162(21.6)$ & $<0.001$ \\
\hline
\end{tabular}

IS New Israeli Shekel (1 NIS 0.25 Euro); PrEP pre exposure prophylaxis 
Most participants indicated that they would prefer to get the PrEP prescription from their primary care provider rather than from AIDS treatment centers or AIDS organizations. In order to implement this PrEP service, dedicated physicians and clinic staff should be trained to respond in a culturally competent and non-stigmatizing way to the upcoming influx of MSM inquiring about PrEP. Providers should be knowledgeable about common high-risk behaviors in the MSM community, possible side effects of the drug, and its proper use.

Men in our study were asked to indicate their willingness to take PrEP in different potential efficacies as were presented in the questionnaire and at various costs. As expected, higher efficacy and lower cost increase the willingness. Interestingly, participants were more sensitive to the price of the drug rather than its potential efficacy, and halving the price doubled the willingness. In order to reduce the financial constraints and decrease the incentive of MSM to purchase PrEP online, an alternative route should be generated to provide the drug to men who are unable to bear the copayment. Since our study was performed, new information shows the effectiveness of PrEP is higher than 95\%, as it was originally presented to the participants during the study and the price has been reduced. Therefore, the willingness to take PrEP may be underestimated in our study. As we learned from the recent COVID-19 outbreak [21], the use of PrEP may also be reduced during a period characterized by a social atmosphere of insecurity and apprehension.

Public health interventions aiming to promote PrEP among MSM should consider the knowledge gap found among MSM in this study. This study showed that MSM who had not heard of PrEP were younger, non-Jewish, and had a lower level of education. These MSM may also be less exposed to other safe-sex messages and, therefore, can be reached through smartphone applications, which are cheap, widespread, and allow users to maintain anonymity.

Oral PrEP is an effective biomedical method used to prevent HIV infection, adding to condoms and other harm reduction strategies. As the success of the treatment depends on appropriate use and adherence, health providers should be attentive to possible structural barriers that exist for MSM to access the treatment, such as negative stigma of being promiscuous, lack of perceived HIV risk, difficulties in accessing clinics or paying for the drug, inability to manage the multiple follow-up visits or low tolerability of the medication [22]. Several strategies have been proposed to achieve a large scale of PrEP coverage among MSM. First, more family doctors should advise PrEP to MSM who are at high sexual risk. Doctors should be informed of the updated followup recommendations and the possible side effects of the medication. In addition, PrEP should be integrated with STD screening clinics and HIV testing sites. Second, an alternative "on demand" course can be proposed to MSM who are discouraged by the daily pill burden. The alternative drug regimen has at least comparable effectiveness to daily use [23]. Third, PrEP education material should be distributed by community-based organizations and health insurers to empower MSM to take control over their health. These interventions should address possible stigma related to PrEP use. This may be especially valid among marginalized or sub-urban MSM populations or minorities, such as Arab [24]. Fourth, financial barriers should be eliminated. The Israeli AIDS task force and a pharmaceutical company provide free PrEP to MSM who face financial difficulties. Last, medical insurers should deploy policy-level solutions to promote sexual health in community clinics and HIV prevention interventions. This includes training programs for physicians, encouraging them to raise the issue of sexual orientation while taking medical history and discuss possible consequences of high-risk sexual risk behavior with their patients. In order to promote HIV-prevention, medical insurers should also encourage family physicians to propose PrEP proactively and reassure that those who are using PrEP are being followed-up.

\section{Limitations}

This is the first study conducted in Israel that assesses knowledge of and willingness to use PrEP, yet it is subject to several limitations. First, this study included a convenience sample in Israel, making the representativeness of MSM who participated in the study difficult to assess and limiting its generalizability to other countries. Of note, the population which responded appeared to be engaged in high-risk sexual behavior, thus making our assumptions appropriate for high-risk MSM. Second, all collected data were self-reported by participants and may be subject to bias, including social desirability and recall bias. In order to minimize these biases, a computeradministered questionnaire was used, reducing pressure for favorable reporting while ensuring that most questions in the study pertained to the last 6-12 months.

\section{Conclusions}

PrEP awareness was high in this study and $44.8 \%$ of participants indicated that they were willing to take PrEP, especially MSM who were involved in high-risk sexual behaviors. The social acceptability of PrEP in Israel is likely to have increased since its approval in September 2017. The findings of this study support Israel's current policy to allow MSM who are at high sexual risk to take PrEP under medical follow-up. However, further studies should evaluate the awareness of and willingness to use 
PrEP over time and assess the actual uptake of PrEP, its positive consequences, and possible adverse outcomes (e.g., greater exposure to STDs).

\section{Supplementary Information}

The online version contains supplementary material available at https://doi. org/10.1186/s13584-021-00500-x.

Additional file 1. Study Questionnaire.

\section{Acknowledgements}

The authors thank Ms. Vicki Cohen for her logistic help with this study.

\section{Authors' contributions}

ZM initiated the study, collected and analyzed the data, wrote the first draft; GS wrote the study questionnaire and helped in interpreting writing the final versions; IL commented in data analysis and contributed in writing the final versions. All authors read and approved the final manuscript.

\section{Funding}

The study was partially funded by Gilead, Israel.

\section{Availability of data and materials}

The datasets used and/or analyzed during the current study are available from the corresponding author on reasonable request.

\section{Declarations}

Ethical approval and consent to participant

The study was reviewed and approved by the Institutional Review Board of the Sheba Medical Center in Tel Hashomer, Israel.

\section{Consent for publication}

The data were collected anonymously through an electronic survey.

\section{Competing interests}

The authors declare that they have no competing interests.

\section{Author details}

${ }^{1}$ Tel Aviv Department of Health, Ministry of Health, 12 Ha'arba'a Street, Tel Aviv, Israel. ${ }^{2}$ School of Health Sciences, Ashkelon Academic College, Ashkelon, Israel. ${ }^{3}$ School of Social Work, Faculty of Social science, Tel Aviv University, Tel Aviv, Israel. ${ }^{4}$ AIDS and STD Unit, Sheba Medical Center, Tel Hashomer, Israel.

${ }^{5}$ Faculty of Medicine, Tel Aviv University, Tel Aviv, Israel.

Received: 3 January 2021 Accepted: 7 November 2021

Published online: 06 December 2021

\section{References}

1. Mor Z, Dan M. The HIV epidemic among men who have sex with menbehaviour beats science. EMBO Rep. 2012;13:948-53.

2. Hess KL, Crepaz N, Rose C, et al. Trends in sexual behavior among men who have sex with men (MSM) in high-income countries, 1990-2013: A systematic review. AIDS Behav. 2017;21:2811-34.

3. Grant RM, Lama JR, Anderson PL, et al. Preexposure chemoprophylaxis for HIV prevention in men who have sex with men. N Engl J Med. 2010;363:2587-99.

4. Desai M, Field N, Grant R, McCormack S. Recent advances in pre-exposure prophylaxis for HIV. BMJ. 2017;359:j5011.

5. United States Food and Drug Administration. FDA approves first drug for reducing the risk of sexually acquired HIV infection. 2012. https://aidsi nfo.nih.gov/news/1254/fda-approves-first-drug-for-reducing-the-risk-ofsexually-acquired-hiv-infection. Accessed 15 June 2019
6. World Health Organization. Guidelines on oral pre-exposure prophylaxis (PrEP) for serodiscordant couples, men and transgender women who have sex with men at high risk for HIV: Recommendations for the use in the context of demonstration projects. Geneva: WHO; 2012.

7. Centers for Disease Control and Prevention: US Public Health Service: Preexposure prophylaxis for the prevention of HIV infection in the United States-2017 update: A clinical practice guideline. 2018. https://www. cdc.gov/hiv/pdf/risk/prep/cdc-hiv-prep-guidelines-2017.pdf. Accessed 15 June 2019.

8. Chemtob D, Mor Z, Harel N, et al. HIV infection among men who have sex with men in Israel: a 35-year epidemiological and clinical overview, 1981-2015. BMC Public Health. 2019;19(1):747.

9. Peng P, Su S, Fairley CK, et al. A global estimate of the acceptability of pre-exposure prophylaxis for HIV among men who have sex with men: a systematic review and meta-analysis. AIDS Behav. 2018;22:1063-74.

10. European Centre for Disease Prevention and Control/WHO Regional Office for Europe. HIV/AIDS surveillance in Europe 2018-207 data. Stockholm: ECDC; 2018.

11. Smith DK, Pals SL, Herbst JH, et al. Development of a clinical screening index predictive of incident HIV infection among men who have sex with men in the United States. J Acquir Immune Defic Syndr. 2012;60:421-7.

12. Frankis J, Young I, Flowers P, McDaid L. Who will use pre-exposure prophylaxis (PrEP) and why? Understanding PrEP awareness and acceptability amongst men who have sex with men in the UK-A mixed methods study. PLoS ONE. 2016;11:e0151385.

13. Kesler MA, Kaul R, Myers T, et al. Perceived HIV risk, actual sexual HIV risk and willingness to take pre-exposure prophylaxis among men who have sex with men in Toronto, Canada. AIDS Care. 2016;28:1378-85.

14. Spinner CD, Hanhoff N, Krznaric l, et al. 2016 PREP attitudes in Germany: High awareness and acceptance in MSM at risk of HIV. Infection. 2018:46:405-8.

15. Grov C, Whitfield TH, Rendina HJ, et al. Willingness to take PrEP and potential for risk compensation among highly sexually active gay and bisexual men. AIDS Behav. 2015;19:2234-44.

16. Montaño MA, Dombrowski JC, Dasgupta S, et al. Changes in sexual behavior and STI diagnoses among MSM initiating PrEP in a clinic setting. AIDS Behav. 2019;23:548-55.

17. Jenness SM, Weiss KM, Goodreau SM, et al. Incidence of gonorrhea and chlamydia following human immunodeficiency virus pre-exposure prophylaxis among men who have sex with men: a modeling study. Clin Infect Dis. 2017;65:712-8.

18. Mor Z, Maoz S, Sheffer R. The European men who have sex with men, internet study (EMIS-2017): Israeli reoprt. Tel Aviv, Israel, 30 Decemebr 2020. Electronic access: S-2017_National-Report_IL.pdf (sigmaresearch. org.uk).

19. Mor Z, Davidovich U. Same-sex sexual attraction, behavior and practices of Jewish men in Israel and the association with HIV prevalence. AIDS Care. 2016;28(Suppl 1):64-7

20. Nic Lochlainn L, O'Donnell $K$, Hurley $C$, et al. Using data from a behavioural survey of men who have sex with men (MSM) to estimate the number likely to present for HIV pre-exposure prophylaxis (PrEP) in Ireland, 2017. Euro Surveill. 2017;22(48):17-00768.

21. Shilo G, Mor Z. COVID-19 and the changes in the sexual behavior of men who have sex with men: results of an online survey. J Sex Med. 2020;17(10):1827-34

22. Sullivan PS, Mena L, Elopre L, Siegler AJ. Implementation strategies to increase PrEP uptake in the south. Curr HIV/AIDS Rep. 2019;16(4):259-69.

23. Molina JM, Charreau I, Spire B, et al. Efficacy, safety, and effect on sexual behaviour of on-demand pre-exposure prophylaxis for HIV in men who have sex with men: an observational cohort study. Lancet HIV. 2017;4(9):e402-10.

24. Mor Z, Grayeb E, Beany A. Arab men who have sex with men in Israel: Knowledge, attitudes and sexual practices. HIV Med. 2016;17(4):298-304.

\section{Publisher's Note}

Springer Nature remains neutral with regard to jurisdictional claims in published maps and institutional affiliations. 